\title{
Reacción psicológica ante la experiencia de abuso sexual extrafamiliar en padres de niños abusados
}

\section{Psychological reactions in parents of children who have suffered extra-familiar sexual abuse}
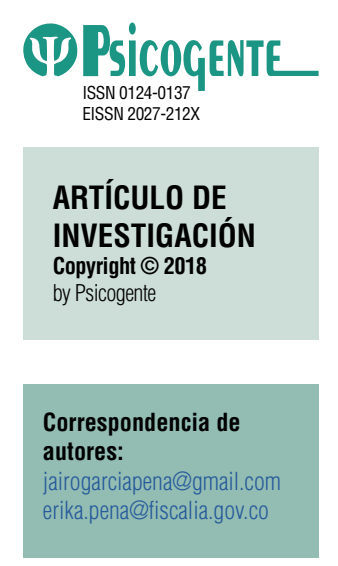

Recibido: 02-02-17 Aceptado: 25-09-17 Publicado: 01-07-18

\author{
John Jairo García Peña (iD \\ Universidad de Antioquia, Medellín, Colombia. \\ Erika Lucia Peña Londoño (iD \\ CAIVAS, Fiscalía, seccional Medellín, Colombia.
}

\section{RESUMEN}

Objetivo: Analizar las reacciones psicológicas en padres de niños que han sufrido la experiencia de abuso sexual extrafamiliar-ASE, realizado en el Centro de Atención Integral a Víctimas de Abuso Sexual-CAIVAS, de la Fiscalía, seccional Medellín, Colombia.

Método: El estudio se realizó desde un enfoque cualitativo, histórico hermenéutico, con metodología fenomenológica. Para la recolección de la información, se hicieron entrevistas a diez padres de familia, nueve madres y un padre, quienes acudían a proceso judicial por denuncia penal del evento ASE; estos se eligieron de manera selectiva, desde el archivo de noticia criminal que recibe el área de psicología, y participaron de manera libre, a partir de firma de consentimiento informado. El análisis de la información generada se realizó a través de una categorización y codificación relacional.

Resultados: Los más relevantes indican traumas transgeneracionales y sentimientos difusos como padres cuidadores, que dificultan la forma de asumir sus funciones parentales e impiden que sean agentes protectores para sus hijos.

Conclusión: Se evidenció que el abuso sexual extrafamiliar tiene implicaciones directas en los padres y que su intervención exige abordar todo el sistema familiar, ya que cuando una familia se expone a este evento, la dinámica se problematiza, dificultando la protección del hijo y la capacidad de afrontamiento de este, ante una amenaza de este tipo.

Palabras clave: abuso sexual extrafamiliar, víctimas, reacciones psicológicas, parentalidad.

\section{Abstract:}

Objective: This paper is the result of a research whose objective was to analyze psychological reactions in parents of children who have suffered extra-familial sexual abuse, ASE; this research was conducted at Centro de Atención Integral a Víctimas de Abuso Sexual - CAIVAS - Prosecutor office, in Medellin, Colombia.

Method: A qualitative, historical hermeneutic approach was used, through a Phenomenological methodology; for data collection, ten parents taken from criminal new files, and who were received through a free and informed consent signature by psychology area, were sampled, as follows : nine mothers and one father, who attended to judicial process for criminal proceeding related to ASE event . In order to analyze the information, a categorization and relational codification, which allowed us to know psychological reactions from parents whose children were victims of sexual abuse, were made.

Results: The most relevant results are trans-generational traumas and diffuse feelings, such as caregiver parents, for whom to assume their parental functions is difficult and prevent them to be their children protectors.

Conclusion: As a conclusion, it was evident that extra-familial sexual abuse has direct implications on their parents, as well as its intervention, demands to approach the whole family system; because when a family is affected by this event, the dynamics are problematized, not only making the child protection difficult for parents, but also for helping the child develop coping skills with a threat of this type.

Keywords: extra-familial sexual abuse, victims, psychological reactions, parenting.

Cómo citar este artículo (APA):

García Peña, J. J. \& Peña Londoño, E. L. (2018). Reacción psicológica ante la experiencia de abuso sexual extrafamiliar en padres de niños abusados

Psicogente 21(40), 378-402. https://doi.org/10.17081/psico.21.40.3079 


\section{INTRODUCCIÓN}

Las cifras de abuso sexual denunciado en Colombia se incrementan a diario, lo cual puede indicar que la conciencia social acerca de la importancia de la denuncia de esta problemática psicosocial ha ido creciendo. La Fiscalía General de la Nación indicó que, en el primer semestre de 2015, se recibieron denuncias de 4448 imputaciones por delitos sexuales, de los cuales 3188 fueron ataques contra menores de edad. Por su parte, el Centro de Atención Integral a Víctimas de Abuso Sexual-CAIVAS, de la Fiscalía, seccional Medellín, reporta 4589 casos de abuso sexual durante los últimos 5 años (Redacción Ámbito Jurídico, 2015).

Llama la atención la prevalencia de la modalidad de abuso sexual intrafamiliar en comparación con el extrafamiliar. Por ejemplo, las cifras reportadas por el CAIVAS muestran que en 2015 fueron 113 los casos de abuso sexual donde el presunto agresor fue un familiar o conocido; mientras que los casos de abuso sexual extrafamiliar-ASE, es decir, donde el presunto abusador no presenta ningún tipo de parentesco o reconocimiento directo con el niño víctima del abuso sumaron 32 (Redacción Ámbito Jurídico, 2015).

Precisamente, el objeto de este artículo es el abuso sexual de tipo ASE, que, en términos de antecedentes considerados durante los últimos 10 años, y en particular, sobre el tema de los padres frente al ASE, evidencian un vacío en la producción científica en comparación con los estudios realizados con padres en el abuso sexual de tipo Intrafamiliar-ASI. En esta vía, se encuentran investigaciones que abordan la disminución del malestar emocional de las víctimas del ASE, favoreciendo la intervención en crisis, pero que no involucran directamente a los padres (Grosz, Kempe \& Kelly, 2000; OMS, 2002).

El ASE se caracteriza porque se comete por fuera del núcleo familiar o es perpetrado por una persona no perteneciente al mismo, generando un daño multidimensional en todo el sistema familiar en el cual los padres se configuran como víctimas no culpables (Echeburúa y Guerrica, 2000; Arriagada y Thiers, 2005; Babatsikos \& Miles, 2015). Las investigaciones reportan que en muchos de los casos se presenta un factor común, y es el secreto familiar o el ocultamiento de información por parte de la víctima y su familia, por el temor a que no se le crea en su confesión, en su entorno familiar y por el rechazo social, que puede acompañarse de posibles sanciones legales que afectan a la familia. 
En este mismo orden, la mayoría de los estudios sobre el ASE ponen su atención en el niño o niña víctima, pero existe poco material encontrado sobre las consecuencias en los demás miembros de la familia, quienes, como afirman McCourt, Peel \& O'Carroll (1998), se denominan "las víctimas secundarias" o "las víctimas invisibles" del abuso sexual.

Estos últimos autores resaltan el tratamiento sobre la situación de abuso en las víctimas, las conductas de los agresores y sus motivaciones en la realización del hecho abusivo, pero también reiteran que son pocos los estudios dirigidos tanto a la comprensión y al tratamiento de las consecuencias relacionadas con los padres no agresores de víctimas de abuso sexual, lo que los ubica no solo en el lugar de "víctimas secundarias" sino en el de "víctimas secundarias olvidadas" (McCourt et al., 1998).

Por otra parte, Manion, Mcintyre, Firestone, Ligezinska, Ensom \& Wells (1996) afirman que esta problemática debe presentarse como una oportunidad para evaluar el ajuste parental en los padres de niños víctimas de ASE, y precisan que no atenderles ni reconocer su implicación como padres, puede traumatizar a todo el sistema familiar, debido al alto malestar emocional experimentado tras el evento, lo cual puede ocasionar un pobre funcionamiento familiar y llevarlos a una baja satisfacción en su rol parental.

En esta medida, es necesario ampliar la atención más allá del niño víctima e intervenir a las familias golpeadas por este evento, a fin de proponer acciones integrales, que repercutan en los niños víctimas y en toda la familia.

Según plantean Finkelhor, Ormord, Turner \& Hamby (2005), el núcleo familiar en el ajuste psicológico de un ASE, es fundamental y debe considerarse en la detección de familias en riesgo y en la evaluación de casos de abuso. Por ello, Kim \& Kim (2005) afirman que no se debe dejar a un lado a la estructura familiar en la recuperación de la situación abusiva.

En un sentido similar, Elliot \& Carnes (2001) y De Gregorio (2007) señalan cómo los planteamientos teóricos y las medidas asistenciales se han centrado, en su mayoría, en el ámbito individual, es decir, en la víctima, e indican que, para abordar la problemática del ASE desde una comprensión integradora, hay que plantearse preguntas sobre la manera en que los padres representan psicoafectivamente la experiencia de abuso sexual extrafamiliar de sus hijos y sus reacciones psicológicas ante esta experiencia. 
Por todo lo anterior, es necesario conocer en profundidad desde la Psicología cuál es la experiencia vivenciada por padres de familia, e incluso con la familia extensa, puesto que son víctimas también de la situación de ASE sufrida por sus niños. En este marco, nuestra pregunta a investigar fue: ¿cuáles son las reacciones psicológicas de los padres de niños que han sufrido la experiencia del abuso sexual extrafamiliar?

\section{MÉTODO}

\subsection{Diseño}

La investigación se orientó desde el enfoque cualitativo, histórico hermenéutico, y a partir de una metodología fenomenológica. Según Hernández, Fernández y Baptista (1997), la lógica de la metodología cualitativa ofrece una valiosa alternativa para el estudio de fenómenos humanos complejos, porque hace posible comprender los significados de las experiencias intersubjetivas e intrasubjetivas de los participantes en el ambiente natural, a partir de sus experiencias, perspectivas, opiniones y significados, es decir, desde la forma en que los participantes perciben subjetivamente su realidad.

Este enfoque hace posible una mayor comprensión y síntesis de los eventos, tomando como eje central la postura subjetiva y las vivencias de los participantes (Creswell, 1998). Desde la fenomenología es fundamental al respecto interpretar, descubrir y describir la realidad de los participantes, desde sus propias nociones, creencias, que se manifiestan en sus mismas acciones discursivas. En este caso, con esta metodología, se tiene el propósito de conocer las vivencias de los padres en el abuso sexual extrafamiliar de un hijo.

La fenomenología posibilita un tipo de diseño de acción en el que la realidad es construida por las personas involucradas en la situación que se estudia, y aunque las versiones de los mundos sean personales, se pueden estimar las construcciones subjetivas que se parecen y tienen puntos en común (Stake, 1998; Parra y Jaime, 2002).

\subsection{Instrumentos}

La técnica de recolección de información utilizada fue la entrevista semiestructurada; la cual, según Flick (2004), se basa en una guía de contenidos o tópicos en la que el entrevistador puede introducir preguntas adicionales, a fin de profundizar en los conceptos $u$ obtener mayor información acerca del tema explorado. Dicha entrevista pretende establecer un diálogo entre 
investigador y participante enmarcado en un clima empático y abierto a la emergencia de la información, y evitar la inclusión de preguntas y respuestas cerradas.

La entrevista cualitativa se da de manera flexible, de manera que el participante pueda expresarse libremente acerca de sus experiencias. Este tipo de entrevistas, según Grinnell \& Unrau (2007), se fundamenta en una guía general de contenido y el entrevistador posee toda la flexibilidad para manejarla. En este sentido, se propone una conversación temática, de manera sensible y cotidiana en el entendimiento de que a través de este tipo de entrevista se puede entender la naturaleza del fenómeno de los padres frente al ASE de su hijo.

\subsection{Participantes}

La población entrevistada se conformó con diez padres de niños y niñas del Centro de Atención Integral a Víctimas de Abuso Sexual-CAIVAS de la Fiscalía seccional Medellín, quienes estaban en proceso judicial por denuncia penal del evento de ASE; estos padres se eligieron de manera selectiva, desde el archivo de noticia criminal que recibe el área de psicología, de modo que se les invitó previamente y decidieron participar de manera libre, a partir de la firma de consentimiento informado.

De estos diez padres de familia de niños víctimas ASE, nueve (9) son madres y uno (1), padre; asimismo, respecto a sus hijos, ocho (8) son niñas y dos (2) son niños. Se tomaron como criterios de selección: padres de niños que hayan tenido experiencia de abuso sexual extrafamiliar; padres que convivan con sus hijos y asuman el rol de cuidadores y que sean mayores de edad. No se tomaron como sujetos de investigación padres menores de edad, que tuvieran una condición de discapacidad mental, que no convivieran con sus hijos o que, aun cumpliendo los criterios de selección, sus hijos hubieran sido víctimas de otro tipo de abuso sexual.

\subsection{Procedimiento}

El proceso de comunicación e información pretendido con el instrumento permitió ampliar temas emergentes, captando de forma amplia la experiencia vivida por cada uno de los participantes. De esa forma, fue posible comprender en contexto la experiencia del padre que ha pasado por la experiencia de ASE; y por el lado del investigador, se obtuvo una vasta visión para que, más 
allá del evento traumático, ir a los aspectos psicoafectivos y relacionales que subyacen a dicha experiencia.

Por tanto, las entrevistas abordaron aspectos relacionados con las reacciones psicológicas, como representaciones afectivas de los padres de niños víctimas de ASE, a partir de las categorías de análisis que buscan responder a la pregunta de esta investigación.

Las entrevistas se realizaron de forma individual, mediante un encuentro personal con cada participante, en un tiempo aproximado de 45 minutos. En materia de consideraciones éticas es de señalar que durante el proceso de recolección de la información con cada padre entrevistado, se administró un consentimiento informado, en el cual se hacía explícita la confidencialidad, el uso exclusivo con fines académicos de la información y los agradecimientos a cada participante; asimismo, ofrecía realizar una devolución sobre dicha participación a partir de los hallazgos encontrados, la cual se hizo con cada uno de los participantes. Es de anotar también que en esta investigación, desde el procedimiento planteado, no se presentó ningún tipo de conflicto de interés.

Por último, cabe aclarar que las entrevistas se grabaron en audio y posteriormente fueron transcritas en su totalidad para su análisis.

\subsection{Análisis de datos}

Se realizó por medio de una codificación relacional, como primer paso de sistematización de la información generada en las entrevistas. A partir de las categorías de análisis que guiaron las entrevistas, se incluyó la fragmentación, conceptualización y articulación analítica desde la contratación de la pregunta con los objetivos de investigación; procedimiento del cual se derivaron nuevos conceptos y categorías emergentes. Para efectos del informe de investigación, se realizó inicialmente una codificación abierta, a partir de los aportes dados por los participantes durante las entrevistas, asignándoles códigos conceptuales; luego se llevó a cabo una codificación axial, por medio de la cual se seleccionaron las categorías más relevantes, estableciendo relaciones entre las categorías; finalmente, se utilizó la codificación selectiva, por medio de la cual se buscó alcanzar un mayor nivel de abstracción, así como identificar las categorías centrales en torno a las cuales se agruparon las demás, y que dieron cuenta de los resultados de investigación. 
El análisis realizado en esta investigación se hizo de manera simultánea a la recolección y sistematización de los datos, lo cual obedece al diseño metodológico propio de la investigación cualitativa, donde la construcción de realidades múltiples como enfoque posibilitó el análisis de los datos, acorde con los contenidos y narrativas de los participantes, es decir, de la forma cómo hablan, viven, sienten, piensan e interactúan los padres de niños víctimas del abuso sexual. Como herramienta fundamental, el análisis de contenido permitió encontrar y relacionar categorías claves, que respondían a la pregunta de investigación que direccionó este estudio.

\section{RESULTADOS}

Las reacciones psicológicas en este estudio se conciben como las manifestaciones expresadas y narradas por los padres de familia escuchados respecto a la experiencia de abuso sexual extrafamiliar de sus hijos, concretamente, desde la forma como la vivencian, perciben y significan. En este apartado se pretende explicar el contenido psicológico de dichas reacciones, desde la exposición de las categorías de análisis que han guiado esta investigación.

Por tanto, a continuación se enuncian las categorías de análisis y posteriormente los contenidos que las condensan, en las unidades de significado que contienen la esencia de estos resultados. Es de señalar que en cada categoría se destacan las situaciones que se presentaron de forma recurrente como vivencia o representación del fenómeno, en la mayoría de los diez padres de familia participantes.

\subsection{Listado de categorías de análisis}

- Abuso transgeneracional y secreto familiar

- Reacción psicológica después del abuso sexual

- Cambios parentales a posteriori

- Intervención psicológica y Recursos de afrontamiento

\subsubsection{Abuso transgeneracional y secreto familiar}

El abuso transgeneracional se entiende como una experiencia vivida y repetida de forma patente o implícita bajo las características de un secreto, con base en la cual se construyen unas formas particulares de crianza a partir de la desconfianza, el miedo, así como apegos excesivos y desorganizados, sin regulación emocional alguna. 
En esta investigación se encontró este concepto de manera reiterada. Así, de los diez casos participantes, ocho reportan que en su historia de vida también, como sus hijos, habían sufrido abuso sexual, y en todos estos se repite esta situación. Por tanto, el abuso transgeneracional se observa como parte del contexto específico del abuso sexual, que se presenta en una misma familia repetidas veces, de manera que en varias generaciones se ha victimizado a los hijos, padres, tíos y muchas veces hasta a abuelos o la tercera generación ascendente, observándose como un fenómeno que determina la historia familiar y la crianza.

En específico, la participante número 2 reporta: Yo también fui abusada (comienza a llorar) y cómo le parece que mi mamá me contó ahora que ella también, cuando era chiquita. A mí no me gusta hablar de eso.

En este fragmento puede verse una vivencia que se perpetúa desde el pasado sin ser nombrada porque emocionalmente no ha sido tramitada.

Así mismo, la participante 7 refiere: Sí, yo lo viví, sino que nunca me ha gustado hablar de eso porque eso es algo que afecta mucho. De pronto hay algunas que sí sienten rabia, pues, así como está y verla así, me hace recordar todo eso y eso me tiene muy afectada.

Al respecto se podría decir que las vivencias no han sido elaboradas debido a lo doloroso que resulta no solo el retornar su propia experiencia abusiva, sino también revivirla con la experiencia de su propio hijo, lo cual afecta todo el sistema ante el temor de que el secreto familiar salga a la luz.

Se evidencia, además, que a los diez entrevistados se les dificulta asumir actitudes protectoras hacia sus hijos. Es decir, de alguna forma inconsciente permiten que sus hijos sean objetivados sexualmente por un abusador.

Un relato ilustrativo es el siguiente:

Es que la niña ha sido dos veces abusada, la primera vez fue con el hermanito. Yo a la niña la arreglé, la bañé, la vestí toda linda, la dejé jugando con los jugueticos en la sala, cuando la niña no está en la sala, pues iah! se me subió para la plancha. Entonces subí cuando ya vi al hermanastro, que estaba abusando de la niña (E\#6). 
Los aportes de los participantes en esta categoría conllevan a pensar en cómo puede relacionarse el abuso de un hijo con el que han sufrido previamente sus padres.

Por otra parte, en los discursos también emergieron hitos fundamentales en las vidas de las madres, como: tener una historia difícil en la niñez por la crianza que tuvieron, abusos sin resolver, situaciones sin contar debido al secreto familiar. Vivencias estas de alto impacto emocional y de gran repercusión en sus vidas, que de igual forma se encuentran en el terreno de lo no resuelto.

En esta materia se cuenta, por ejemplo: Cuando a mí me pasó, yo nunca hice nada, me quedé callada porque ahorita no es como antes, antes eso era como a escondidas, los padres tapaban todo, yo lo viví, sino que nunca me ha gustado hablar de eso, porque eso es algo que afecta mucho (E\#4).

En este último aspecto del secreto, el hecho de no querer hablar del tema, como reportan ocho de los participantes, es relevante: es algo que se ve como un continuo y que demuestra la repercusión en los significados del abuso y del consecuente rol parental.

La vivencia de estos padres, en relación con el hecho de haber pasado por una experiencia de abuso sexual, y en concreto por un desconocido de la familia, igual que en el caso de sus hijos, lleva a pensar que los reiterados actos de abuso que aparecen en la vida de estas familias no son gratuitos; pues desde esta perspectiva, al haber pasado por una agresión sexual, un padre pareciera no contar con elementos intrapsíquicos suficientes para proteger a sus propios hijos, incrementando las posibilidades de volver a ser victimizado en su siguiente generación. Así, el secreto delictivo se impone nuevamente, por temor a expresar el evento y lo que podría generar el reconocerse como víctima.

Es así como estos padres recuerdan su infancia como una etapa difícil en su vida, que los retorna a su estado anterior. Un ejemplo de ello es expresado de la siguiente manera: Yo tengo un recuerdo de infancia, la verdad es que en mi caso nunca se denunció, era mi vecino, yo a lo último me quedé callada y nunca dije nada. Para mí ha sido lo más grotesco que me ha pasado en la vida y más que me quedé callada y, ahora, que soy grande, pienso que uno chiquito sí es bobo porque me quedé callada (E\#9). 
Esa situación, manifestada en la esfera de lo inconsciente, se evidencia de manera traumática como un efecto en las dinámicas familiares, esto es, en el transcurrir de las generaciones, como una tendencia a guardar el secreto de haber vivido una experiencia de abuso sexual, tanto en el espacio intrapsíquico como en el interpersonal; aspecto este relevante, para entender lo psicopatológico de las experiencias de abuso sexual extrafamiliar y lo patógeno de los secretos familiares.

\subsubsection{Reacción psicológica después del abuso sexual y posición subjetiva del padre}

Los sentimientos y reacciones psicológicas de los padres que se generan a partir de una experiencia de abuso sexual de su hijo son diversos y dan cuenta de su representación parental, es decir, de la forma cómo sienten que están respondiendo a su función de crianza.

En los diez entrevistados, se observa que las reacciones psicológicas más frecuentes que experimentan los padres son: primero, la culpa, por haber fallado en sus capacidades parentales, $y$, segundo, los sentimientos ambivalentes hacia el niño y el agresor:

Nosotros salíamos de la casa a las cuatro y media de la mañana y llegábamos a la siete u ocho de la noche, por temprano que llegáramos, entonces esa culpabilidad era de que ella todo el día estaba sola, llegaba a estudiar y sola. Entonces, ella no tenía como quien la defendiera en ese momento (E\#3).

Otro aporte al respecto:

Es que yo la miraba y me daba rabia, cómo es que se le sirvió a ese desgraciado. Yo desde que lo conocí le vi las intenciones, él es un mal hombre (E\#7).

Estos relatos también tienen que ver con los bajos niveles de parentalización propios de estos casos. En ellos, los padres no logran ver el abuso que ocurre en sus hijos, muchos lo dejan ver como consecuencia de la falta de tiempo para compartir y cuidarles, además de la pobre comunicación con los hijos por el mismo motivo del tiempo. Otros culpan el exterior, su propio hijo o la situación de vida; otros, sin embargo, solo lo reprimen y lo justifican, casi que normalizando el acto abusivo como parte de la vida, pues, ellos también pasaron por esta situación y lo perciben y relacionan con sus hijos de la misma forma. 
Por ejemplo, manifiesta otra madre: Es muy duro, pero uno puede seguir adelante, es peor tanto alboroto que se hace hoy en día, vea en las que me tienen (E\#4).

Estos padres se ven bloqueados incluso al llanto y expresión del malestar de sus hijos. Aun sabiendo y sospechando, se quedan en la esfera del recuerdo de su propia vivencia traumática, sin pasar a la acción como cuidadores en su rol actual.

Se configura así la hipótesis de una falla en la forma de asumir las funciones parentales, lo cual da cuenta de una incapacidad, por su misma historia, de asumir la función de cuidador y protector de su hijo.

En el mismo sentido, se puede observar la relación o correspondencia con historias de abuso preexistentes: La niña una vez se puso a decirnos al papá y yo, llorando, es que ustedes no me creen porque él pasa y los saluda y pasa así normal y se hace el bobo para que ustedes no me crean a mí, entonces ahí fue cuando más se me metió la intriga, o sea, yo no dormía pensando en eso: así mismo me pasó a mí (E\#7).

Cuando se hace conciencia de esa falla de las funciones, aparece lo que se ha observado en torno a la culpa, un sentimiento desagradable de no haber estado allí, de volver a permitir que pase lo que ya pasó.

Pero, como también se ha señalado, en principio este sentido se configura en el ámbito de lo inconsciente, que desde las barreras del secreto y en los modos de crianza aprendidos en familia perpetúan la situación: Cuando ella me contó, me sentí que no les había dedicado todo el tiempo que necesitaban, porque si esto pasó, si esto lleva tiempo, era para yo, ya haberme dado cuenta, entonces sentí que me faltó tiempo (E\#1).

En esta misma vía, se puede ver que existe una especie de sentimientos comúnmente encontrados en los padres de niños abusados extrafamiliarmente. Uno de ellos es la culpa arraigada sobre las exigencias culturales del rol protector materno, según el cual la madre debe estar todo el tiempo muy pendiente de las actividades que rodean a su hijo.

En el relato de cinco de los padres entrevistados, estos perciben negligencia parental en su propio rol, a raíz de no haber anticipado ese evento traumático, que como ellos, sus hijos tuvieron que pasar. 
Asimismo, en términos de reacciones psicológicas, los padres sienten rabia y tristeza en forma entremezclada, lo cual se afianza al pensar en ese suceso traumático como sentimientos que aparecen unidos y que no encuentran su sitio:

Estoy muy perturbada, confundida, siento de todo, sentimientos encontrados, demasiados, porque me está trabajando lo del abuso sexual, lo del pasado, iba por el camino, iba por el túnel, casi saliendo del túnel, me tuve que devolver otra vez. No estaba muy pendiente de la psicóloga, sino de la niña, yo la llamé pero no contesta, nadie me va a entender cuando hablo del túnel, es que cuando las personas que hemos pasado por abuso sexual se nos acaba medio mundo, uno trata de salir, busca opciones, y me propuse a salir, pero en estos días vi que tocaron a mi niña, entonces retrocedí, para eso necesitamos ayuda, los padres necesitamos ayuda, porque sé que muchas mujeres lo necesitan (E\#6).

Pero, también en los padres que no han sido objeto de abuso con anterioridad se puede ver un sentimiento de incapacidad o de impotencia frente a este evento, debido a las afectaciones emocionales que implica, pero también por las exigencias y cánones culturales, que dictan reglas acerca de cómo debe ser la protección de los padres hacia sus hijos.

En este sentido, pueden expresarse ideas repetitivas en los participantes, que, ante un caso de abuso sexual, no logran activar las funciones parentales desde lo que se espera: Las cosas están ahí, pero uno no las ve sino cuando pasan, ahí es cuando uno dice: carajo qué paso acá, o sea, es que el sentimiento de uno sentirse bobo, de uno sentirse impotente, porque mire a mi hija lo que le pasa (E\#9).

Del mismo modo se pudo observar la sensación de un dolor insuperable, evidenciándose en las dificultades en los mecanismos de recuperación del suceso acontecido, ya que no solo es el dolor del hijo, sino también el de padre, sea por lo ocurrido o por lo que retorna, a partir de su propia historia y las trasposiciones de la representación con la emoción.

Esto causa una confusión aparente y transitoria: No, no, culpable, no, no me sentí culpable, no me sentí culpable, vuelvo y te repito, tristeza, pues porque vuelve y se repite, pero no, culpa no, me pregunto por qué, por qué, por qué se repite (sonríe) (E\#5). 
A propósito, se puede rescatar lo que dice Abbagnano (2007, p.253): “El dolor constituye la característica fundamental de la vida emotiva, más precisamente la negativa, casi siempre interpretada como una señal o indicación de carácter hostil, contra la situación en la cual se encuentra el ser vivo".

De esta manera, las representaciones que estos padres y madres construyen de los demás sugieren un monto importante de miedo y angustia frente a la vida y frente a su rol como padres, desde sus representaciones, proyectadas sobre la situación que están viviendo con sus hijos. Cabe preguntarse, entonces, si su dolor es por ellos mismos, por el hijo o por ambos, así: No y no, completamente no, con ella que es mi niña menor, siempre fue uno de mis grandes miedos con una situación de estas por lo que yo sentícuando era niña por lo que me pasó y por lo que me quedé callada, o sea, por todo. En realidad no pensé que reaccionaria así y ahora es llorar y llorar, es como si me sudaran los ojos, no puedo parar de llorar (E\#9).

\subsubsection{Cambios parentales a posteriori}

En los reportes de las entrevistas, se destacan los siguientes sentimientos más comunes de los padres de niños víctimas de ASE: dolor, rabia, impotencia, miedo, desprecio, tristeza, ganas de matar al agresor, vergüenza, ira, culpa, odio, desilusión, angustia, desconfianza, frustración e ideas suicidas. Reacciones psicológicas posevento, que repercuten de una forma muy particular en los vínculos y función parental de estos padres, así como en las relaciones intersubjetivas de los miembros del sistema familiar.

Así, cada una de estas reacciones psicológicas, que surgen a partir de una experiencia de abuso por parte de un hijo, afecta los vínculos entre los miembros que se encuentran inmersos. Las carencias afectivas que entran a suplir terceros, como abuelos, tíos o personas allegadas a la familia, se tornan cada vez más complejas y necesarias para mitigar el dolor y la culpa a partir del suceso, al apartarse los padres del sufrimiento del otro (hijo) y ocuparse de su propia historia de abuso transgeneracional.

De allí que sea frecuente y preferible, en las percepciones de los padres, reportar un sentimiento de ignorancia, incredulidad y negación con respecto al abuso sexual de un hijo, como un saber no sabido, como si solo fuera un presentimiento, una sospecha, un conocimiento de que algo sucede, pues algo les impide asumir conscientemente lo que sucede con ese otro: 
Destrozada, rabia, eso no se entiende nada en ese momento, o sí debe haber cosas que uno entiende, pero provocaba matar a esa persona, aunque yo soy una persona que no sería capaz de hacerle daño a nadie, pero en ese entonces sí (E\#6).

Además, los padres fantasean venganzas como intentos de tramitar el dolor y la confusión emocional que vivenciaron, como lo explicita este padre, único varón de los entrevistados: Lo primero que quisiera hacer es tener esta persona (el agresor) que hizo lo que hizo con estas dos niñas, en especial con mi hija, quisiera tenerlo al frente, quisiera hacer lo que la autoridad y la justicia no hará con él, quisiera tenerlo yo y hacerle lo mismo que le hizo a mi hija, quisiera encontrarlo, cogerlo, golpearlo, picarlo, yo soy capaz de picarlo, porque me duele, me duele, y estoy herido por lo que le hizo a mi hija; sería capaz de muchas cosas (E\#8).

Es como una repetición, que se encripta y reaparece cuando no se quiere saber al respecto: Es que es muy difícil, es como si los volviera a sentir el doble o el triple, impotencializados por la actitud de mi hija, como yo le dije ahora, no sé qué esperar, una niña devastada o una niña inconsciente de la situación, porque cuando yo vi a la niña, yo de verdad lo sentí, yo sentíque de verdad necesitaba cuidado (E\#9).

Por todo esto, es de vital importancia la intervención que se pueda brindar a las víctimas secundarias del abuso sexual extrafamiliar, a fin de que, a su vez, adquieran herramientas para ayudar a las víctimas primarias a organizar la experiencia a partir de la intervención (Barudy \& Dantagnan, 2005). Así: A raíz de lo que pasó con el niño, pues, yo he tratado de estar más pendiente de ellos, si antes los llamaba, ahora los llamo más, yo he hablado mucho con el niño y yo le digo: mire, no puede volver a esa unidad, no puede volver a estar cerca de ese señor, siempre que salga diga para dónde va, estoy con un amiguito. Pues, bueno, entonces como he estado tan pendiente de él, entonces me dice: mami tráigame una cosita, entonces yo trato de llevarles alguna cosita, todas las noches, cualquier cosa, pero entonces ya, yo no sé hasta qué punto uno debe cambiar, porque entonces ya el niño trata de manipular la situación, pues, me ve un poquito culpable (E\#10).

\subsubsection{Intervención psicológica y recursos de afrontamiento}

Sobre este aspecto, se puede observar que a mayor frecuencia, situación crónica e intensidad del abuso, se presentan más dificultades en la función de parentalidad, en términos de negligencia paterna, culpa, odio, impotencia, 
sentimiento de evasión e incredulidad. Inclusive presentándose la negación o rechazo del acto o la rabia, sentimientos que detonan sintomatologías psicológicas en todo el núcleo familiar implicado. Así lo nombra una madre:

Uno siempre se siente culpable, yo me sentía culpable, porque ese día la dejé con una muchacha que todavía estaba joven y salió para la calle, o sea, uno tiene que mirar con quién dejar los hijos cuando va a ir a trabajar, uno nunca piensa que esas cosas le van a pasar a un hijo y a la final que yo me esté callada, uno no se debe quedar callado de estas cosas, por miedo; yo me demoré para hablar, por miedo, que lo van a matar a uno, porque siempre lo amenazan a uno (el agresor), casi siempre hay amenazas, pero se trata de mi hija y se trata de cuántas personas también puedan ser víctimas (E\#5).

En este sentido, cuando las madres callan su propio abuso sexual, les resulta difícil aceptar el abuso del otro, en este caso su propio hijo; ya que no consiguen estar atentos para evitar este hecho, ni perciben los riesgos que el niño corre. De este modo, los hijos de padres con historias de abuso pueden llegar a ser más vulnerables a situaciones de diversas violencias y muy puntualmente de las violencias sexuales, tal como lo muestra la experiencia analizada anteriormente.

Desde el punto de vista de esas vivencias, se destaca, desde los recursos subjetivos con los que cuente el padre, la exacerbada búsqueda de acciones que intentan reparar el daño: Sí, yo lo viví, yo tuve sed de venganza, iba a coger un fusil y lo iba (sonríe). Sí, sí, es terrible, sentí odio en mi corazón, mucho odio, cuando chiquita no sentí esto porque yo lo recordé ya grande, ya grande me llené de odio, de rabia, quería suicidarme porque había quedado en embarazo, quería suicidarme, nunca lo intenté, solo lo pensé, pero sí, es terrible, es muy terrible lo que uno siente (E\#5).

Algunos padres que han vivido este tipo de situación recurren a la medicación psiquiátrica u otros medios para afrontar el dolor y lo que está sucediendo: $Y$ yo en este momento estoy tomando fustina (fluoxetina), para estar tranquila, llevo años tomándola, porque eso me tranquiliza, me pone un poquito dura, por eso cuando no me la tomo, no puedo olvidarlo (E\#6).

Como ya se ha resaltado, también se afecta la vivencia del mismo cuerpo y su funcionamiento, creando esquemas repetitivos y pensamientos obsesivos desde el orden del cuerpo y la limpieza, y es que la idea de la violencia, la mayoría de las veces, no ocurre de forma unidireccional, ya que ella actúa 
en la vida de sus víctimas de forma sistemática. No se puede tomar un abuso sexual de forma aislada, individual, porque el entramado social de las personas se interrelaciona, formando un conjunto.

En este sentido, una evaluación psicológica tomada a tiempo aumenta la probabilidad de fortalecer los recursos de afrontamiento, permitiendo, por ejemplo, interrumpir la cadena de abusos, identificar las consecuencias y, por tanto, dirigir la intervención de forma adecuada, mediante la expresión y la elaboración de las respuestas emocionales (Pereda, 2009).

Esto se evidencia en el siguiente aporte: Para serle sincera, yo lo daba como un sentimiento guardado, yo lo daba por guardado, eso pasó cuando yo era niña, o sea yo daba por hecho de que esto ya pasó. Lo que pasa es que se me vino encima anoche con lo de la niña, pero hasta ayer por la noche, yo estaba tranquila, yo llevaba años sin pensar en eso, es como si fuera un archivo y mi memoria hubiera dicho, esto está muy viejo y puf, yo me acuesto en la noche y pienso en que me sentí vulnerada y que me sentíatacada y que sentí muchísimo miedo. Esos recuerdos y sentimientos volvieron anoche (E\#9).

De esta forma, la intervención psicológica facilita esclarecer el recuerdo desde la palabra. Es así como, en ese espacio de intervención, además de la palabra y las representaciones emocionales, se hacen presentes respuestas afectivas que posibilitan la vivencia de una adultez sana:

Nunca le puse atención a eso, sino que lo pensé más por el lado de cómo ella es la más grandecita, pensé yo, de pronto allá hay unas niñas como más pequeñas y lo hacen. Entonces ella de pronto se está valiendo de eso, porque ella a veces me llegaba con palabras muy de bebé como las que decía, ella está con una niña especial, entonces yo dije, de pronto lo está asociando con todo lo que la niña hace y no le vi como mucha malicia, porque yo dije, no, pues si ella de pronto se me está devolviendo, porque las otras niñas de pronto lo están haciendo (E\#7).

En definitiva, los niveles de conciencia aumentan luego de una intervención psicológica en este tipo de sucesos. Las habilidades parentales que en un principio se evidenciaron en déficit, empiezan a desarrollarse más adecuadamente.

Es que es muy difícil. La actitud de mi hija, como yo le dije ahora, no sé qué esperar, una niña devastada, cuando yo la vi, yo de verdad lo sentí, sentí que de verdad necesitaba cuidado, protección, y ahí me va a tener (E\#9). 


\section{DISCUSIÓN}

El abuso sexual es un tema que en sí implica lo infantil, porque conlleva unos comportamientos de poder sobre un niño o niña que se halla en situación de vulnerabilidad física, mental, social y comportamental por su misma condición evolutiva.

Como se ha definido desde los mismos antecedentes de este estudio, el abuso sexual se especifica según los ámbitos de ocurrencia y niveles del abuso. De ahí, que se hable de abuso sexual en la tipología intrafamiliar y abuso sexual en la tipología extrafamiliar. En ambos terrenos se encuentran comprometidos no solo el niño o niña víctima, sino también su entorno inmediato: padres, hermanos y familia extensa (Holmes \& Slap, 1998; Kim \& Kim, 2005; Echeburúa y Guerrica, 2000; Arriagada y Thiers, 2005; Babatsikos \& Miles, 2015; Grosz, Kempe \& Kelly, 2000).

Justamente, ello da pie a esta investigación desde el interés de profundizar el conocimiento acerca de las implicaciones de la experiencia de abuso sexual, en términos de reacciones psicológicas de los padres y la familia.

En esta vía, se retoman, a continuación, las categorías de análisis para mostrar los resultados que hicieron posible responder a la pregunta que guió este estudio, en relación con los antecedentes y los conceptos referenciados.

\subsection{Abuso transgeneracional y secreto familiar}

El abuso transgeneracional, se ha conceptualizado entre otras definiciones como el trauma transgeneracional debido a la vivencia repetida entre las distintas generaciones de una familia. En tal sentido, según autores como Glaser \& Frosh (1993); Rotenberg (2014); Kilroy, Egan, Maliszewska \& Sarma (2014), consultados en los antecedentes, la experiencia abusiva parece comportarse como una cadena intergeneracional de traspaso.

Más concretamente, Glaser \& Frosh (1993) se refieren a este fenómeno como "encadenamiento materno" del abuso sexual entre madres e hijas/os. Pues, aun cuando se reconozca que quienes abusan sean otras personas fuera del núcleo familiar, hay algo desde las prácticas de crianza, que se transmite y empuja, a que se viva el abuso en los hijos de madres que, a su vez, tienen una historia de abuso sexual.

Al respecto, Sampson (1991) plantea que las vivencias afectivas no resueltas se perpetúan desde el pasado pero sin ser nombradas, porque según la 
indicación emocional no han sido tramitadas a pesar del paso de los años. Es decir, la representación psicoafectiva se muestra en la emoción angustiosa del llanto ante el recuerdo de la situación vivida, lo cual genera y exacerba las respuestas psicológicas de estos padres.

De esta manera, el abuso y la propensión al mismo hacen que los niños parezcan imanes de abusadores, pero en realidad la situación está mediada por un entramado subjetivo e intersubjetivo dentro de una sociedad dada, donde el subsistema familiar juega un papel de réplica de situaciones conscientes o inconscientes de generación en generación, que en algunos casos se encuentra en la esfera del tabú (López, 1994).

\subsection{Reacción psicológica después del abuso sexual y posición subjetiva del padre}

Como se viene planteando, las reacciones psicológicas en este estudio se comprenden como las manifestaciones narradas por parte de los padres de familia, sobre la forma cómo vivencian, perciben y significan la experiencia de abuso sexual extrafamiliar de sus hijos; reacciones que de forma empírica se exteriorizan mediante las emociones diversas que se generan a partir de una experiencia de abuso sexual que involucra a un niño. Es importante señalar que dichas reacciones psicológicas conservan un hilo conductor en su mayoría, según cómo vivan su propia representación parental. Al respecto, en los antecedentes, se pudo rastrear que para distintos autores, entre ellos Echeburúa y Corral (2006) y Finkelhor (2008), la reacción emocional que tome el padre o cuidador ante la revelación del hijo, influirá en las consecuencias que el trauma opere en el presente y vida futura de este niño.

Por esta razón, es muy importante conocer y prestar atención a las reacciones psicológicas de los padres ante este tipo de eventos (Magalhaes, Gimeniz \& Moreira, 2009). En nuestro contexto, pareciera que la reacción psicológica de los padres que más aparece, desde lo encontrado en seis de los relatos de los entrevistados, es la no credibilidad en la narración del niño o niña, lo cual también podría verse como una defensa psíquica del adulto cuidador, debido a la impotencia y al sentimiento de haber fallado como padre, puesto que al haberse desarrollado el abuso fuera del hogar, se manifiesta una culpa en el padre que genera pocas estrategias de afrontamiento en el marco de su función parental.

En este último campo, investigaciones como la realizada por Arriagada \& Thiers (2005) describen la escasa literatura e investigaciones empíricas 
referidas al impacto que produce la situación abusiva en los padres. No por ello, resulta relevante poner la mirada en dichos padres que -como el infante- sufren un impacto psicológico.

Echeburúa y Corral (2006) confirman lo anterior y plantean que la recuperación del abuso sexual infantil dependerá del grado de culpa que siente el niño, así como de las estrategias de resiliencia de que disponga la víctima y sus padres, quienes al igual que la víctima sufren las consecuencias del hecho abusivo.

Por otro lado, esos sentimientos suelen imposibilitar a los padres para asumir la responsabilidad moral de hacerse cargo de lo que conlleva social y jurídicamente un acto como el abuso. Se demostró, en efecto, que, en la mayoría de las denuncias reportadas, los padres se enteran del abuso de un hijo por un tercero ajeno a la familia; asimismo, la tendencia a ignorar como padres el acto abusivo del hijo, surge como un mecanismo defensivo para protegerse de las consecuencias que acarrea este suceso, como son el señalamiento social y la acusación de negligencia parental, por no haber estado allí para evitar el hecho, entre otras.

\subsection{Cambios parentales}

Del Campo (2002) trae a colación el concepto de cripta, para dar cuenta de los contenidos psíquicos inconscientes por parte de los padres, que pueden estar marcados por el funcionamiento psíquico de los abuelos o de los ancestros que aun sin ser conocidos los marcó de alguna manera; se trata de ese saber no sabido, que marca una experiencia en la generación siguiente, dejando huellas inconscientes del abuso sexual, como una cadena que permanece en el tiempo.

Estos conceptos de transmisión generacional y cripta guardan una estrecha relación con el trauma que conlleva el abuso sexual, es decir, lo que es innombrable para una generación (padres) es impensable para su descendencia (sus hijos), quienes están condenados, por el secreto familiar, a ser "portadores" de ciertos contenidos psíquicos que se transmiten de generación en generación (Del Campo, 2002).

\subsection{Intervención psicológica y recursos de afrontamiento}

En este campo, es importante destacar la investigación de Echeburúa y Corral (2006), quienes enfatizan que no todas las personas cuidadoras responden de la misma forma ante el abuso. Debido a esto, aunque, como se ha 
expresado, haya asuntos que se puedan generalizar en la forma de afrontar este fenómeno, no todos los padres comparten las mismas características psicológicas y manifiestan sus emociones de forma distinta, en momentos diversos, por ende, se puede decir que el impacto emocional que se genera al respecto es heterogéneo y subjetivo, de modo que cada caso amerita su análisis.

Sin embargo, los mismos autores antes citados han propuesto cuatro variables que aparecen en estas situaciones: el perfil individual del padre (recursos psicológicos, edad, sexo y el apoyo familiar); las características del acto abusivo (haber pasado por una experiencia de abuso sexual, frecuencia del acto abusivo, callar mediante el secreto familiar, el abuso ocurrido en la infancia, entre otros); la relación existente con el abusador (abuso intrafamiliar o extrafamiliar) y, por último, las maneras de enterarse del acto abusivo.

En general, el impacto de las secuelas depende de la frecuencia, duración y consumación de la experiencia, así como del uso de la fuerza y amenazas por parte del agresor o la existencia de una violación mediante acceso carnal a la víctima. El conocimiento claro de estos datos clínicos podría hacer posible que los padres asuman situaciones familiares y de procesos de crianza que les ayude a fortalecer sus recursos de afrontamiento.

En síntesis, el abuso sexual contra un niño genera tantos traumas psicológicos y emocionales para sus víctimas que cuando llegan a la edad adulta, como estos padres, se evidencian secuelas insuperables, que influencian de forma marcada sus respuestas parentales y muy específicamente su reacción ante la historia de abuso de un hijo, quedando, con frecuencia inmersos en los sentimientos de culpa, dolor, impotencia, resentimientos, tristeza profunda, entre otras reacciones psicológicas devastadoras e incapacitantes. Así, conforme destacan los padres de niños víctimas del abuso sexual, ellos siguen incluidos en esa cadena de víctimas afectadas por las violencias sexuales.

\section{CONCLUSIONES}

En primer lugar, se puede concluir que el análisis de los recursos de afrontamiento de los participantes se encuentra en un nivel de desarrollo básico. $\mathrm{Si}$ bien estos padres y madres son personas funcionales en las demás áreas de su vida, a la hora de entenderse con el abuso sexual extrafamiliar de sus hijos aparecen defensas psicológicas muy básicas como la negación, la justificación y la proyección. A partir de la escucha atenta, lo anterior permitió reconocer 
que la mayoría de los participantes en este estudio (ocho de diez) también habían sufrido abuso sexual en su infancia y que en seis de ellas, debido a la influencia social, al tabú y a la tradición arraigada del secreto familiar, nunca se hizo público el evento. De esta manera, esta transmisión de lo no dicho, que pasa a ser de la esfera de lo inconsciente, se perpetúa generacionalmente, convirtiéndose en reacciones psicológicas básicas y defensas primarias, hasta que se haga posible un espacio como el ofrecido en este estudio para que sea expresado y elaborado de una forma más adecuada. En la misma línea, su entorno inmediato se ve afectado, de tal manera que el secreto familiar resulta actuando como un predictor de abusos generacionales.

En segundo lugar, las reacciones psicológicas de los padres que han tenido hijos abusados extrafamiliarmente, están teñidas por las representaciones que tienen de sí mismos, de sus objetos de cuidado y del sistema familiar. En este sentido, prevalecen imágenes disociadas entre la vivencia del pensamiento y del afecto. En estas circunstancias, el cuerpo se vive de forma generalmente desagradable lo cual trae como consecuencia que los padres no logren observar o reconocer los indicadores de abuso que presentan sus hijos, y el afecto termina reprimiéndose o expresándose en forma de angustia, insomnio, y de síntomas psicológicos como inapetencia sexual y el deseo de venganza.

En tercer lugar, cabe decir que las representaciones psicoafectivas de los padres de los hijos abusados extrafamiliarmente han sido poco exploradas, tanto desde la investigación como desde la intervención psicológica. Estas representaciones operan desde lo inconsciente y hacen que las personas actúen de forma mecánica. Así, aparecen elementos de desvalorización del discurso de los hijos y de negación de las situaciones. Además, otro elemento que se representa son los sentimientos de culpa, en los cuales el padre siente responsabilidad en el acto, por no estar pendiente de la manera que socialmente se espera. En el extremo opuesto también puede encontrarse la autojustificación, basada en que las demandas de la vida diaria no permiten al padre cumplir con lo que se esperaba o en la simple negligencia parental.

En este mismo sentido, se destaca que todos estos padres aún se preguntan por el sentido de la experiencia en sus vidas y, por qué hay elementos que se repiten de un modo exacto, incluso por tres generaciones. Una respuesta a ello podría hallarse en el secreto familiar respectivo, que se perpetúa en las representaciones, en las funciones parentales, en la vivencia del cuerpo y en la formas de crianza. 
Finalmente, se encuentran secuelas profundas del abuso y de la reminiscencia en los padres de los niños abusados. Estas secuelas se perpetúan, según lo observado, en la transmisión generacional. De esto depende la forma en que los padres afronten la situación del abuso, la representación que hacen del mismo y sus recursos para ejercer la paternidad.

Por esto la intervención psicológica con estos padres es de vital importancia; el hecho de ofrecer un espacio de comunicación para el intercambio de significados, como fue la entrevista semi-estructurada, operó, en efecto, como un cambio y abrió las puertas a reflexiones subjetivas profundas en los participantes.

\section{RECOMENDACIONES}

Antes de finalizar este trabajo de carácter cualitativo, se recomienda emprender acciones que permitan el avance del tema tanto en lo científico como en lo práctico. En concreto, se recomienda:

Emprender acciones para estudiar el ASE desde un estatuto científico que permita más comprensión del fenómeno y que haga posible, a su vez, una comprensión de orden práctico, para prevenir este tipo de situaciones, desde la intervención de la problemática y el manejo vivencial de la sociedad en general. Desde esta perspectiva de comprensión, serían pertinentes investigaciones futuras, como por ejemplo, acerca de la vivencia en niños en comparación a la de las niñas; o, sobre la incidencia y prevalencia en un entorno familiar afectado por este evento, en relación a las repercusiones del secreto familiar y la transmisión intergeneracional como efecto en las costumbres, comportamientos y prácticas de crianza de dicho contexto familiar.

Asimismo, es claro que el abuso sexual perpetrado por hombres ha sido estudiado con más profundidad como se evidencia en la literatura revisada. Pero, no se han hecho averiguaciones avanzadas respecto al abuso sexual perpetrado por mujeres. Esta inquietud surge a raíz de una de las entrevistas, donde se insinúa esta posibilidad en el relato intergeneracional de una de las participantes.

Desde el punto de vista representacional, es importante asumir elementos de análisis más profundos, utilizando esquemas de evaluación estandarizados como el test de relaciones objetales y los test proyectivos, que pueden dar lugar a evaluaciones más extensas y generalizantes. De esta manera, se va transitando el camino del modelo cualitativo desde otros puntos de 
vista, mediante los cuales se pueda profundizar la complejidad de la realidad evaluada.

En cuanto a la intervención, se recomienda brindar atención psicológica a los padres y madres víctimas secundarias de abuso sexual extrafamiliar. En estos casos, como se ha planteado, las víctimas de ASE presentan una carga emocional fuerte que no siempre logran tramitar, la repetición de esos elementos literalmente actúan casi como un cáncer en los sistemas familiares y personales que los contienen.

A la hora de atender psicológicamente a las víctimas secundarias de ASE, se recomienda evaluar su historia vincular, puesto que en ella aparecen elementos que brindan pistas para brindar un adecuado manejo de la situación y prevenir eventos de este tipo.

En el mismo sentido de la prevención del abuso sexual, se recomienda a las instituciones que ofrecen servicios para la familia y la niñez, desarrollar protocolos de evaluación del riesgo para el abuso sexual externo. En el mercado existen muchos protocolos de evaluación del riesgo de abuso sexual pero no para la tipología externa. Es de vital importancia, además, que en las citadas instituciones haya cursos de concientización y formación para apoyar a los padres y madres a evaluar y fortalecer sus prácticas de crianza y sus funciones de parentalización. Como se encontró en el estudio, hay un déficit importante en su capacidad como padres cuidadores.

También sería interesante que se formen grupos de apoyo emocional para los padres de niños víctimas de abuso sexual, puesto que desde la misma vivencia, resignificada, se pueden enfocar intervenciones que fortalezcan la autoestima y el autoconcepto de las víctimas y la comunidad que los rodea.

Finalmente, por las implicaciones de alto impacto de esta problemática psicosocial, se recomienda continuar con investigaciones al respecto, a fin de aportar al conocimiento y prevenir a nivel familiar, social e institucional la aparición de este tipo de problemáticas y fomentar su tratamiento adecuado.

NOTA DE AUTORES: Artículo resultado de la investigación "Representación psicoafectiva y reacción psicológica ante la experiencia de abuso sexual extrafamiliar en padres de niños abusados".

\section{REFERENCIAS}

Abbagnano, N. (2007). Diccionario de filosofía. São Paulo (SP): Martin Fontes. 
Arriagada, A., y Thiers, C. (2005). Diseño, implementación y validación de una intervención psicoeducativa grupal para padres no agresores de niños víctima de abuso sexual (Tesis de grado). Universidad de Chile, Santiago de Chile. Disponible en http://repositorio.uchile.cl/handle/2250/113530

Babatsikos, G. \& Miles, D. (2015.). How parents manage the risk of child sexual abuse: a grounded theory. Journal of child sexual abuse, 24(1), 55-76. https://doi.org/10. 1080/10538712.2015.981352

Barudy, J. \& Dantagnan, M. (2005). Los buenos tratos a la infancia: parentalidad, apego y resiliencia. Barcelona: Gedisa.

Creswell, J. (1998). Qualitative inquiry and research design. Choosing among five traditions. USA: Sage.

De Gregorio, A. (2007). El abuso sexual infantil y la mala praxis psiquiátrica y psicológica. Recuperado de https://es.scribd.com/document/332835781/El-abuso-sexual-infantil-y-la-mala-praxis-psiquiatrico-psicologica-pdf

Del Campo, A. (2002). Abuso sexual a menores: historia, concepto y tipos de abusos. Salamanca: Universidad de Salamanca.

Echeburúa, E. y Guerrica, C. (2000). Abuso sexual en la infancia: víctimas y agresores. Barcelona: Ariel.

Echeburúa, E., y Corral, D. (2006). Secuelas emocionales en víctimas de abuso sexual en la infancia. Cuadernos de medicina forense, (43-44), 75-82. Disponible en http:// scielo.isciii.es/scielo.php?script=sci_arttext\&pid=S1135-76062006000100006

Elliott, A. \& Carnes, C. N. (2001). Reactions of nonoffending parents to the sexual abuse of their child: a review of the literature. Child maltreatment, 6(4), 314-331. https://doi.org/10.1177/1077559501006004005

Finkelhor, D. (2008). Abuso sexual al menor. Causas, consecuencias y tratamiento psicosexual. México DF: Editorial Pax México.

Finkelhor, D., Ormond, R., Turner, H. \& Hamby, S. L. (2005). Measuringpoly-victimization using the juvenile victimization questionnaire. Child abuse \& neglect, 29(11), 1297-1312. https://doi.org/10.1016/j.chiabu.2005.06.005

Flick, U. (2004). Introducción a la investigación cualitativa. Madrid: Morata.

Fondo de las Naciones Unidas para la infancia (UNICEF). (2014). Informe anual 2014 (Colombia). Recuperado de http://www.unicef.com.co/reporte_anual_2014/ reporte_anual_2014.pdf

Glaser, D. \& Frosh, S. (1993). Child Sexual Abuse. Second Edition. Londres: MacMillan Press.

Grinnell, R. \& Unrau, Y. (2007). Social workresearch and evaluation: foundations of evidence based practice. New York: Oxford University Press.

Grosz, C., Kempe, R. \& Kelly, M. (2000). Extrafamilial sexual abuse: treatment for child victims and their families. Child abuse \& neglect, 24(1), 9-23. https://doi org/10.1016/S0145-2134(99)00113-1

Hernández, S., Fernández, C., y Baptista, L., (1997). Metodología de la investigación. Bogotá: Panamericana.

Holmes W, \& Slap G. (1998). Sexual abuse of boys: definition, prevalence, correlates, sequelae, and management. Journal of the American medical association, 280 (21), 1855-1862. http://dx.doi.org/10.1001/jama.280.21.1855

Kilroy, J., Egan, J., Maliszewska, A. \& Sarma, M. (2014). "Systemic trauma": the impact on parents whose children have experienced sexual abuse. Journal of child sexual abuse, 23(5), 481-503. https://doi.org/10.1080/10538712.2014.920458

Kim, H. \& Kim, H, (2005). Gender differences in delinquent behavior among korean adolescents. Child psychiatry and human development, 35(4), 325-345. https:// doi.org/10.1007/s10578-005-2691-1 
López, F. (1994). Los abusos sexuales de menores. Lo que recuerdan los adultos. Madrid: Ministerio de asuntos sociales.

Magalhaes, Q., Gimeniz, M. \& Moreira, M. (2009). Abuso sexual infantil: percepción de las madres frente al abuso sexual de sus hijas. Revista latino-americana de enfermagem, 17(4). http://dx.doi.org/10.1590/S0104-11692009000400011

Manion, G., Mcintyre, J., Firestone, P., Ligezinska, M., Ensom, R. \& Wells, G. (1996). Secondary traumatization in parents following the disclosure of extrafamilial child sexual abuse: initial effects. Child abuse \& neglect, 20(11), 1095-1109. https:// doi.org/10.1016/0145-2134(96)00098-1

McCourt, J., Peel, C. \& O'Carroll, P. (1998). The effects of child sexual abuse on the protecting parent $(\mathrm{s})$ : identifying a counselling response for secondary victims. Counselling psychology quarterly, 11(3), 283-299. http://dx.doi.org/10.1080/09 515070.2017.1336077

Organización mundial de la salud (OMS). (2002). Informe mundial sobre la violencia y la salud. Washington, D.C: Organización Mundial de la Salud.

Parra, H., y Jaime, H. (2002). Investigación cualitativa y participativa: un enfoque histórico-hermenéutico y crítico social en psicología y educación ambiental. Medellín: UPB. Disponible en http://portalapp.mineducacion.gov.co/drupalM/?$\mathrm{q}=$ node $/ 2035$

Pereda Beltrán, N. (2009). Consecuencias psicológicas iniciales del abuso sexual infantil. Papeles del psicólogo, 30(2), 135-144. https://doi.org/10.23923/pap. psicol2017.2826

Redacción Ámbito Jurídico. (23 de abril de 2015). Fiscalía revela cifras de delitos sexuales contra menores. Ámbito jurídico. Recuperado de https://www.ambitojuridico.com/BancoConocimiento/Penal/fiscalia-revela-cifras-de-delitos-sexuales-contra-menores

Rotenberg, E. (2014). Parentalidades interdependencias trasformadoras entre padres e hijos. En E. Rotenberg, Parentalidades interdependencias trasformadoras entre padres e hijos. Buenos Aires: Mejora.

Sampson, J. A. (1991). ¿Fantasía o fantasma?. Revista de Psicoanálisis, 3, 47-54. Disponible en http://www.intersubjetividad.com.ar/website/articulop.asp?i$\mathrm{d}=246$ \& idioma $=$ \&idd $=6$

Stake, E. (1998). Investigación con estudio de casos. España: Ediciones Morata. autor o el licenciante.

\section{(c)) BY}

